\title{
UK-based real-time lymphoproliferative disorder diagnostic service to improve the management of patients in Ghana
}

\author{
Elizabeth Parkins • Roger G. Owen • \\ George Bedu-Addo • Ohene Opare Sem • Ivy Ekem • \\ Yvonne Adomakoh • Imelda Bates
}

Received: 26 April 2009/Accepted: 28 April 2009/Published online: 9 July 2009

(C) Springer-Verlag 2009

\begin{abstract}
The objective of the study was to evaluate the feasibility of a UK-based real-time service to improve the diagnosis and management of lymphoproliferative disorders (LPDs) in Ghana. Adult patients reporting to hospital with a suspected LPD, during a 1 year period, were prospectively enrolled. Bone marrow and/or lymph node biopsies were posted to the Haematology Malignancy Diagnostic Service (HMDS), Leeds, UK and underwent morphological analysis and immunophenotyping. Results were returned by e-mail. The initial diagnoses made in Ghana were compared with the final HMDS diagnoses to assess the contribution of the HMDS diagnosis to management decisions. The study was conducted at the two teaching hospitals in Ghana-Komfo Anokye, Kumasi and Korle Bu, Accra.
\end{abstract}

E. Parkins $(\bowtie)$

Royal Cornwall Hospital Trust,

Truro TR1 3LJ, UK

e-mail: lizparkins@nhs.net

R. G. Owen

HMDS Laboratory, St James's Institute of Oncology,

Beckett Street,

Leeds LS9 7TF, UK

G. Bedu-Addo $\cdot$ O. O. Sem

Komfo Anokye Teaching Hospital

and School of Medical Sciences,

Kwame Nkrumah University of Science and Technology,

P. O. Box 1934, Kumasi, Ghana

I. Ekem · Y. Adomakoh

Department of Haematology, Korle Bu Teaching Hospital,

Accra, Ghana

I. Bates

Tropical Haematology, Liverpool School of Tropical Medicine, Pembroke Place,

Liverpool L3 5QA, UK
Participants comprised 150 adult patients ( $\geq 12$ years old), 79 women, median age 46 years. Bone marrow and lymph node biopsy samples from all adults presenting with features suggestive of a LPD, at the two teaching hospitals in Ghana, over 1 year were posted to a UK LPD diagnostic centre, where immunophenotyping was performed by immunohistochemistry. Molecular analysis was performed where indicated. Diagnostic classifications were made according to international criteria. Final diagnosis was compared to the initial Ghanaian diagnosis to evaluate discrepancies; implications for alterations in treatment decisions were evaluated. Median time between taking samples and receiving e-mail results in Ghana was 15 days. Concordance between initial and final diagnoses was $32 \%$ (48 of 150). The HMDS diagnosis would have changed management in $31 \%$ (46 of 150) of patients. It is feasible to provide a UK-based service for LPD diagnosis in Africa using postal services and e-mail. This study confirmed findings from wealthy countries that a specialised haematopathology service can improve LPD diagnosis. This model of Ghana-UK collaboration provides a platform on which to build local capacity to operate an international quality diagnostic service for LPDs.

Keywords Lymphoproliferative disorders · Ghana . Diagnostic service $\cdot$ Lymphoma $\cdot$ Leukaemia

\section{Introduction}

Understanding the genetic and molecular hallmarks of lymphoproliferative disorders (LPDs) has led to many new and highly efficacious therapeutic regimes. Accurate pathological diagnosis and classification according to standard criteria are essential first steps in determining appropriate 
treatment strategies [1]. Accurate diagnosis of LPDs is complex and discrepancies of $20-48 \%$ have been reported between LPD diagnoses made by general histopathologists and those made by specialist haematopathologists [2]. Diagnostic criteria use histological, immunological and molecular techniques, combined with an ability to interpret ambiguities associated with these techniques, and such resources are generally not available in countries in subSaharan Africa. However, initiatives such as health insurance schemes mean that treatments for LPDs, such as chemotherapeutic agents and radiotherapy, are increasingly available in poorer countries, but they cannot be targeted effectively because of inadequate capacity to accurately diagnose and categorise LPDs.

LPDs are a heterogeneous group of neoplastic disorders of the immune system, including lymphomas (non-Hodgkin and Hodgkin), myeloma and lymphoid leukaemias. The incidence, epidemiology and clinical presentation of LPDs vary across the world because they are influenced by factors such as genetic predisposition and environment [1,3,4]. This means that knowledge about LPDs is not necessarily transferable between regions and evidence about local LPD patterns is needed to guide service delivery. For example, one of the only prospective studies in sub-Saharan Africa found a 1:3 male/female ratio in 60 patients with chronic lymphocytic leukaemia (CLL) in Nigeria, which is strikingly different to the usual 2:1 ratio seen in CLL in the west [5]. A retrospective study in Kenya of 207 cases of non-Hodgkin lymphoma that presented over 10 years found that $41 \%$ were not properly classified histologically, and only $15 \%$ of 105 evaluable patients were followed up for at least 36 months [6]. It is particularly important for clinical services in subSaharan Africa to have the capacity to diagnose LPDs because they may mimic infectious diseases especially tuberculosis. A review of 129 lymph node biopsies from Jos in Nigeria found that tuberculosis accounted for $48 \%$ of the cases once sarcomas and carcinomas had been excluded [7]. Rising HIV prevalence in Africa means that atypical presentations of lymphoma and infections such as tuberculosis are increasing, and accurate diagnosis of lymphadenopathy will avoid misdiagnosis and inappropriate treatment of infections and LPDs.

The purpose of this study was to assess the feasibility of providing a UK-based real-time diagnostic service for LPDs for two teaching hospitals in Ghana. The ultimate goal is to strengthen the research opportunities and systems in Ghana for investigating LPDs and to improve evidencebased clinical care of LPDs. Collaborating institutions were the Haematological Malignancy Diagnostic Service (HMDS), Leeds, UK, and Komfo Anokye Teaching Hospital (KATH), Kumasi and Korle Bu Teaching Hospital $(\mathrm{KBTH})$, Accra in Ghana. HMDS serves a population of four million through the Yorkshire and Humberside Cancer
Networks and integrates morphological assessment, immunophenotyping (by flow cytometry and/or immunohistochemistry) and molecular techniques to provide a 'onestop' service for diagnosis of haematological malignancies. Results are double authorised by a combination of consultant haematologists, haematopathologists and scientists before release and e-mailed to clinicians via a password-protected website. Better postal and e-mail services between Ghana and the UK mean that it is now potentially feasible to establish an off-site diagnostic service through HMDS as a prelude to transferring the service to the two Ghanaian teaching hospitals.

\section{Methods}

Study setting

The study was based in two teaching hospitals, KATH and $\mathrm{KBTH}$, in the two largest cities in Ghana. Patients were referred to haematology and oncology clinics from within the hospital or from regional and district hospitals and health centres. Patients aged 12 years and above are seen in the adult haematology clinics. Patients with suspected LPDs were usually referred because of leucocytosis, abdominal mass or lymphadenopathy. Prior to this study, patients were investigated and managed using whatever locally available resources they could afford. Histopathology services were available in KBTH. At KATH, fine-needle aspirates were interpreted on site but biopsy samples were sent to be processed in private histopathology laboratories.

To document the existing system for the diagnosis of LPDs, a retrospective audit of the case notes of patients referred to KATH's haematology and oncology clinics in 2004 (i.e. the year prior to the start of the study), with a suspected diagnosis of LPD, was conducted. In order to capture all potential LPD patients, the case notes of all patients with a provisional diagnosis of lymphoma, leukaemia, myeloma, splenomegaly or lymphadenopathy were reviewed, and data were extracted about clinical features and laboratory results using a pre-designed and piloted form.

New diagnostic service 2005-2006

For 1 year from March 2005, patients aged $\geq 12$ years, presenting to the hospitals' haematology departments with features suggestive of a LPD who gave informed consent were enrolled in the study. Suggestive features included B symptoms (fever, weight loss, sweats), lymphadenopathy, hepatomegaly, splenomegaly, lymphocytosis, hypercalcaemia and lytic bone lesions. Patients with splenomegaly were entered only if they also had lymphocytosis, lymphadenopathy or B symptoms or had failed to respond to proguanil 
therapy for hyper-reactive malarial splenomegaly [8]. Locally available blood tests (e.g. blood count, lactate dehydrogenase, urea, creatinine and electrolytes, liver function tests, calcium, erythrocyte sedimentation rate), X-rays, scans and lymph node biopsies were performed as appropriate.

Peripheral blood $(6 \mathrm{ml}), 2 \mathrm{ml}$ bone marrow aspirate and trephine biopsy samples were taken from each patient. Laboratory bench space, a centrifuge, a $37^{\circ} \mathrm{C}$ water bath and freezer space were made available in each hospital for sample processing. Consumables were sourced locally in Ghana apart from eppendorfs, capped test tubes and pastettes.

An unstained bone marrow aspirate slide and trephine biopsy for each patient, as well as the lymph node biopsy samples, were posted to HMDS using United Postal Services. Biopsy samples were fixed in formalin prior to processing. At HMDS, immunophenotyping was performed by immunohistochemistry on the trephine and lymph node blocks. Standard streptavidin-biotin immunoperoxidase methods were used to phenotype tumour cells using panels of markers selected according to the suspected diagnosis (Table 1). Immunophenotyping results were used in conjunction with morphological and histological findings to arrive at a final diagnosis according to WHO criteria [2]. Where relevant, fluorescence in situ hybridisation and polymerase chain reaction analysis were also performed.

Results from HMDS were e-mailed to the Ghanaian clinicians as soon as they had been authorised. Patients were allocated a unique identification number, and all data were entered onto a password-protected database. Only project clinicians had access to patient data.

The initial diagnosis, based on clinical features and locally available tests, was recorded for each patient. The impact of HMDS results on patient diagnosis and hypothetical implications for their management were evaluated by comparing the HMDS diagnosis with the initial diagnosis. Patients were divided into categories depending on whether the initial diagnosis was the same as the final HMDS diagnosis and whether the final diagnosis would have altered patient management.

\section{Ethics approval}

The project received ethical approval from the ethics committees of Liverpool School of Tropical Medicine, Komfo
Anokye Teaching Hospital (Kumasi) and University of Ghana Medical School (Accra). The project was discussed with eligible patients, via an interpreter where necessary, using specifically designed information and consent forms. Consenting patients (and guardians if they were less than 16 years of age) signed or thumb-printed the form.

\section{Results}

Existing LPD services

Case notes were available for 63 of the 80 patients seen at KATH in 2004 with a suspected LPD. The median age was 48 years (range $12-80$ years) and $42(67 \%)$ were female. B symptoms (fever, sweats, significant weight loss) were present in $26(41 \%)$ patients. Forty-four patients $(70 \%)$ had splenomegaly (median $16 \mathrm{~cm}$; range 4-26 cm), $27(43 \%)$ had hepatomegaly (median $6 \mathrm{~cm}$; range 3-16 cm) and 18 (29\%) had lymphadenopathy at presentation. Forty-eight of $63(76 \%)$ diagnoses were made using clinical features alone. Of these, 28 were hyper-reactive malarial splenomegaly and 19 were LPDs (Table 2). There were 11 cytological diagnoses - ten from fine needle aspirates and one from a bone marrow aspirate. Only four diagnoses were made histologically. Immunophenotyping and molecular techniques were not involved in any of the diagnoses.

New diagnostic service 2005-2006

One hundred fifty patients, 117 from KATH and 33 from KBTH, were entered into the study. There were 79 females, and the median age was 46 years (range 12-83 years). Their samples were sent to HMDS in 25 packages. Each package weighed less than $500 \mathrm{~g}$ and cost $£ 21.34$ /package. All parcels arrived intact at HMDS within three to four working days of being posted. Four packages, packed according to IATA regulations, were carried by hand from Ghana to HMDS. The median time between taking bone marrow trephine samples and receiving e-mail results in Ghana was 15 days (range 7-62 days). For lymph node biopsy results, the median time from samples leaving Ghana to results being emailed back was 11 days (range 3-27).
Table 1 Panels of markers used to phenotype tumour cells

\begin{tabular}{ll}
\hline Suspected tumour & Markers \\
\hline Lymphoma & CD3 CD5 CD10 CD20 CD23 CD79 BCL2 BCL6 Ki67 Cyclin D1 \\
Hodgkin lymphoma & CD3 CD15 CD20 CD30 MUM1/IRF4 OCT2 BOB1 \\
Acute lymphoblastic leukaemia & CD1a CD3 CD7 CD10 CD34 CD79 PAX5 tdt \\
Myeloma & CD138 CD56 MUM1/IRF4 kappa lambda \\
Carcinoma & CD45 MNF116 CK7 CK20 \\
\hline
\end{tabular}


Table 2 Case note diagnoses in patients with suspected LPD: 2004 audit

\begin{tabular}{|c|c|c|c|c|c|}
\hline Diagnosis & $\begin{array}{l}\text { Number of patients } \\
\text { with diagnosis }\end{array}$ & $\begin{array}{l}\text { Diagnosis made on } \\
\text { clinical features alone }\end{array}$ & $\begin{array}{l}\text { Diagnosis made on } \\
\text { fine-needle aspirate }\end{array}$ & $\begin{array}{l}\text { Diagnosis made } \\
\text { on biopsy }\end{array}$ & $\begin{array}{l}\text { Diagnosis made on bone } \\
\text { marrow aspirate }\end{array}$ \\
\hline HMS & 28 & 28 & & & \\
\hline NHL & 13 & 6 & 5 & 2 & \\
\hline HL & 5 & & 3 & 2 & \\
\hline LPD & 5 & $5^{\mathrm{a}}$ & & & \\
\hline CLL & 5 & 5 & & & \\
\hline Myeloma/plasmacytoma & 4 & 2 & 1 & & 1 \\
\hline All & 1 & 1 & & & \\
\hline Portal hypertension & 1 & 1 & & & \\
\hline Metastatic malignancy & 1 & & 1 & & \\
\hline Total & 63 & 48 & 10 & 4 & 1 \\
\hline
\end{tabular}

$H M S$ hyper-reactive malarial splenomegaly, $N H L$ non-Hodgkin lymphoma, $H L$ Hodgkin lymphoma, $C L L$ chronic lymphocytic leukaemia, $A L L$ acute lymphoblastic leukaemia

${ }^{a}$ One had had a biopsy, but result was not available

Final diagnoses

Pathological diagnosis was possible in 132 of the 150 patients. Specimens were not obtained in 14 patients due to early death and loss to follow-up; only four patients had inadequate specimens. Sixty-one of the132 patients $(46 \%)$ had a LPD, 42 (32\%) had other diagnoses and 29 (22\%) had splenomegaly for which no cause could be found (Table 3). Of the 61 LPD diagnoses, there were 30 nonHodgkin lymphomas (18 splenic marginal zone, four diffuse large B-cell, three peripheral T-cell, two Burkitt, two follicular and one mantle cell), seven Hodgkin lymphomas (five nodular sclerosing, two mixed cellularity), 12 CLL, five acute lymphoblastic leukaemias (three T cell, two B cell), six myelomas and one NK cell LPD.

Table 4 shows the initial diagnosis or differential diagnosis made in Ghana before the HMDS result was available. The HMDS diagnosis would not have significantly affected management in 104 of 150 patients $(69 \%)$. In 48 patients $(32 \%)$, the initial diagnosis was correct. In a further 16 patients $(11 \%)$, the final diagnosis was different from the initial diagnosis but management would not have been affected. The HMDS results did not affect diagnosis or management in 40 patients $(27 \%)$ because they were lost from the study due to death or lack of data, a problem frequently encountered in this setting (Table 5).

In 46 of 150 (31\%) patients, the HMDS diagnosis would have changed patient management. In half of these patients $(n=23)$, the HMDS diagnosis was part of the initial differential diagnosis but it would not have been possible to make to appropriate treatment decisions without the additional results. In a further 23 patients, the HMDS diagnosis was not part of the initial differential diagnosis. In a third of patients (15 of 46) in whom the HMDS diagnosis would have altered management, the initial diagnosis was based on fine-needle aspirate cytology (Tables 4 and 5).

\section{Discussion}

This study demonstrates that it is feasible to provide a sophisticated UK-based real-time LPD diagnostic service for patients in Ghana using complex haemato-pathological techniques. The service provides an interim solution until

Table 3 Characteristics of study patients

\begin{tabular}{|c|c|c|c|c|c|}
\hline Diagnosis & $\begin{array}{l}\text { Number of patients } \\
\text { with diagnosis } \\
\text { Total }=132\end{array}$ & $\begin{array}{l}\text { Age years median } \\
\text { (range) }\end{array}$ & $\begin{array}{l}\text { Sex } \\
\mathrm{M} / \mathrm{F}\end{array}$ & $\begin{array}{l}\% \text { patients with } \mathrm{B} \\
\text { symptoms at presentation }\end{array}$ & $\begin{array}{l}\mathrm{WCC} \times 10^{9} / 1 \\
\text { median (range) }\end{array}$ \\
\hline Lymphoproliferative disorders & 61 & $58(12-80)$ & $25: 36$ & 93 & 18.4 (1.5-UR) \\
\hline Other ${ }^{\mathrm{b}}$ or unknown diagnoses & 42 & $33.5(12-83)$ & $25: 17$ & 83 & $7.4(2-240)$ \\
\hline Splenomegaly - unknown cause & 29 & $35(12-70)$ & $13: 16$ & 93 & $3.3(1-26.1)$ \\
\hline
\end{tabular}

UR unrecordable

${ }^{\text {a }}$ Eighteen patients had no/inadequate trephine biopsy and have been excluded from the table

${ }^{\mathrm{b}}$ Acute myeloid leukaemia, refractory anaemia with excess blasts, monoclonal gammopathy of uncertain significance, tuberculosis, Rosai Dorfman, other malignancy, reactive marrow 
Table 4 Initial diagnoses made in Ghana
Initial diagnosis

Number of patients

Lymphoproliferative disorder (LPD)
LPD/hyper-reactive malarial splenomegaly (HMS)
Chronic lymphocytic leukaemia (CLL)
Myeloma
Hodgkin lymphoma
Infection/lymphoma
Acute lymphoblastic leukaemia
Metastatic carcinoma
Marginal zone lymphoma (MZL)
HMS
Diffuse large B-cell lymphoma
LPD/other malignancy
CLL/MZL
Tuberculosis
Follicular lymphoma
Rosai Dorfman
Other
No initial diagnosis given

34

23

17

9

9

9

9

7

5

5

4

4

3

2

1

1

2

5

(/) differential diagnosis

Ghana is able to set up its own international standard diagnostic systems. The quality of samples transported to the UK was good, and the turnaround time was 10-15 days. The service depended on a few critical factors: a dedicated team in each hospital, reliable communications between institutions in Ghana and the UK and an efficient clinical coordinator. The coordinator's role was to ensure the quality of samples, process and document specimens, organise transport and receive and disseminate results.

The HMDS service enhanced the capability of the existing service in Ghana to accurately diagnose suspected LPDs. Overall HMDS diagnosis would have changed management in a third of patients, confirmed initial diagnosis in a third, and had no effect on management in the other third. These results are similar to those from a Welsh study, which found that a panel of specialist haematopathologists disagreed with the initial diagnosis, which would have altered subsequent management in $46 \%$ of suspected LPD cases [2]. Our study provided preliminary information about the prevalence of different types of lymphoma presenting to the hospital, which will enable clinicians to make informed and rational choices about treatment regimes. The collaboration between the Ghanaian institutions and HMDS provided an excellent framework for epidemiological studies and for detailed genetic and molecular genetic assessments in selected patients such as fluorescence in situ hybridisation and immunoglobulin gene sequencing [9].

The success of the system was dependent on good record keeping and effective communication systems between the Ghanaian and UK institutions. It would have been unethical to have withheld appropriate treatment once the HMDS

Table 5 Impact of HMDS diagnosis on patient management

\begin{tabular}{llll}
\hline $\begin{array}{l}\text { Group } \\
(N)\end{array}$ & Initial diagnosis & $\begin{array}{l}\text { Effect of final HMDS diagnosis } \\
\text { on patient management }\end{array}$ & Examples \\
\hline $1(48)$ & Correct diagnosis & $\begin{array}{c}\text { Initial diagnosis confirmed; } \\
\text { no alteration in management }\end{array}$ & $\begin{array}{l}\text { Hodgkin disease } \\
\text { Rosai Dorfman }\end{array}$ \\
$2(23)$ & $\begin{array}{c}\text { No firm diagnosis made; correct final } \\
\text { diagnosis included in differential diagnosis }\end{array}$ & $\begin{array}{c}\text { HMD diagnosis altered } \\
\text { management }\end{array}$ & Final diagnosis did not \\
$3(16)$ & $\begin{array}{l}\text { Final diagnosis not included in initial } \\
\text { differential diagnosis }\end{array}$ & $\begin{array}{c}\text { Final diagnosis altered } \\
\text { management }\end{array}$ & NK cell leukaemia \\
$4(23)$ & $\begin{array}{c}\text { Final diagnosis not included in initial } \\
\text { differential diagnosis }\end{array}$ & $\begin{array}{c}\text { Final diagnosis did not } \\
\text { affect management }\end{array}$ & Nasopharyngeal \\
& Patient died, lost to follow-up or & carcinoma Tuberculosis & NR \\
& inadequate/unobtainable sample & & \\
\hline
\end{tabular}

NR not relevant 
diagnosis was known in Ghana so it was not possible to compare outcomes between, for example, patients who did and did not have samples processed in the UK. However, the retrospective audit of lymphoma patients provides an indication of the extent to which patients could be investigated locally and the range and preciseness of diagnoses compared to the recommended international lymphoma classification categories.

There are very few descriptions in the literature of successful collaborations between wealthy and less wealthy countries to improve the diagnosis of haematological malignancies. Ours is the first study to focus on real-time remote diagnosis for patients in Africa with suspected LPDs. The only published collaboration to use remote diagnosis on posted samples was between institutions in the USA and El Salvador. This study aimed to describe clinical and biological features of childhood leukaemia in El Salvador and to develop effective treatment regimens. Between 1994 and 1996, 153 patients were enrolled in the study. Morphology and cytochemistry were performed locally, and bone marrow samples were sent by post or courier to the USA for immunophenotyping, DNA analysis and molecular genetics. The programme was subsequently extended to Honduras and Guatemala and was successful primarily because it had been generated and part-funded by an enthusiastic local team in El Salvador. Problems encountered with the remote analysis of samples included the inability to get repeat samples when results were inconclusive, loss of samples and delays during transit resulting in samples that were inadequate for testing. As the number of samples increased, the techniques and expertise were gradually transferred to El Salvador [10]. In our study, we had a very low incidence of inadequate specimens presumably due to the use of fixed trephine and lymph node biopsies as opposed to fresh bone marrow aspirate or peripheral blood samples.

Two other collaborations to improve the diagnosis and management of childhood leukaemia involved three centres in India linked with one in the USA and a partnership between Nicaragua, Italy and Switzerland [11, 12]. These collaborations provided local training rather than a remote diagnostic service. Confirmation of the diagnosis of nonHodgkin lymphoma was carried out on samples from Kuwait by an expert panel in Germany but this study was retrospective and was therefore not able to directly influence patient management [13].

An improved diagnostic system is only one component in the pathway to improving clinical outcomes for patients with LPDs in sub-Saharan Africa. Our study was limited to the diagnosis of LPDs and was not designed to measure impact on patient outcomes, which are influenced by many factors beyond this diagnostic system. Patients in developing countries often have to pay for diagnostic tests and treatment. This leads to sub-optimal care especially for the poorest. In Africa, treatment protocols need to be tailored not only to affordability but to availability of drugs and supportive therapies such as platelet transfusions and growth factors. Clinicians' response to changes in diagnostic services is also an important determinant of patient outcomes.

As this was a feasibility study, the costs were met from project funds. Before deciding whether or not to scale up a remote diagnostic service, more research is needed to determine the cost benefits from the perspective of both the patient and the health services. Compared to the existing service in Ghana, the benefits to the patients are a more rapid and accurate process to confirm an LPD or alternative diagnosis and the potential to receive the correct treatment with subsequent positive health benefits. For the health service, the benefits would include information about the causes of lymphadenopathy and local epidemiology of LPDs, which would enable better planning and allocation of oncology resources.

If Ghana and other countries in sub-Saharan Africa are to establish effective treatment programmes for LPDs, then the ultimate aim must be to transfer the diagnostic techniques and the skills to interpret them to institutions in Africa [14]. Our feasibility study has demonstrated that it is possible to set up a sophisticated diagnostic service for patients in Ghana with suspected LPDs that enables patients to be classified according to international standards. This model for Ghana-UK collaboration provides a platform on which to gradually build local capacity to operate an international quality diagnostic service for LPDs. The study has shown that transferring specific immunohistochemistry technology for biopsies to Ghanaian institutions should be a priority as this is a robust technique, which provides good diagnostic information about LPDS and conditions that may mimic LPDs. Transfer of technology needs to be accompanied by development of medical, nursing and paramedical expertise in LPDs, enhanced facilities for supportive care for neutropenic patients and reliable, adequate supplies of safe blood components [15].

Cancer is an important cause of morbidity and mortality in countries in sub-Saharan Africa, and new initiatives such as health insurance schemes are driving demand for better quality diagnosis as well as providing more resources to treat cancers. Collaborations between health care workers and scientists in wealthy and less wealthy countries are an excellent way to bridge the inequalities in cancer services that exist between these countries. Developing countries gain access to new techniques for diagnosis, resources to improve patient management and training to enhance skills and morale. Developed countries broaden their knowledge about cancers and are provided with much better opportunities than are available in their own country to reassess what is essential in 
cancer care and what can be adapted and streamlined [12]. Furthermore, establishment of international quality cancer treatment units in developing countries will attract more physicians and researchers and contribute significantly to global knowledge about the clinical, biological and epidemiological aspects of cancer [16].

Authors' contributions EP was responsible for collecting patient samples, coordinated link between institutions in Ghana and the UK and contributed to the design, analysis and write-up of the study (project was part of her MD thesis).

$\mathrm{RO}$ was responsible for coordinating diagnostic process at HMDS and reporting results and contributed to the design, analysis and write-up of the study.

GBA, OOS, IE and YD were the clinicians responsible for identifying and managing study patients and providing clinical information and contributed to the design and write-up of the study.

IB (guarantor) conceived the study, contributed to the design and analysis and was responsible for drafting the paper.

Competing interests All authors have nothing to declare.

Role of the funding source Funding for this study was provided by HMDS, the Liverpool School of Tropical Medicine. EP self-funded her MD. Study sponsors had no role in study design; in the collection, analysis and interpretation of data; in the writing of the report; and in the decision to submit the paper for publication.

\section{References}

1. Swerdlow SH, Campo E, Lee Harris N, Jaffe ES, Pileri SA, Stein H, Thiele J, Vardiman JW (2008) WHO classification of tumours. Pathology and genetics of tumours of haematopoietic and lymphoid tissues. IARC, Lyon

2. Lester JF, Dojcinov SD, Attanoos RL, O'Brien CJ, Maughan TS, Toy ET, Poynton CH (2003) The clinical impact of expert pathological review on lymphoma management: a regional experience. Br J Haematol 123(3):463-468

3. Anderson JR, Armitage JO, Weisenburger DD (1998) Epidemiology of the non-Hodgkin's lymphomas: distributions of the major subtypes differ by geographic locations. Non-Hodgkin's Lymphoma classification project. Ann Oncol 9:717-720
4. Parkin DM, Pisani P, Ferlay J (1999) Global cancer statistics. CA Cancer J Clin 49:33-64

5. Omoti CE, Awodu OA, Bazuaye GN (2007) Chronic lymphoid leukaemia: clinico-haematological correlation and outcome in a single institution in Niger Delta region of Nigeria. Int $\mathrm{J}$ Lab Hematol 29(6):426-432

6. Othieno-Abinya NA, Abwao HO, Maina JM, Nyabola LO, Opiyo A, Njuguna E, Ndege P, Musibi A (2004) Non-Hodgkin's lymphomas at Kenyatta the National Hospital Nairobi in the 1990's. East Afr Med J 81(9):450-458

7. Obafunwa JO, Olomu IN, Onyia NJ (1992) Primary peripheral lymphadenopathy in Jos, Nigeria. West Afr J Med 11(1):25-28

8. Bedu-Addo G, Bates I (2002) Causes of massive tropical splenomegaly in Ghana. Lancet 360:449-454

9. Stephens E, Bates I, Bedu-Addo G, Opare Sem K, Ekem I, Dei Y, O'Connor SJM, Evans PAS, Rollett RA, Owen RG (2007) Tropical splenic lymphoma: detailed clinico-pathological assessment in 19 Ghanaian patients. Br J Haematol 137S1:19

10. Bonilla M, Moreno N, Marina N, de Reyes G, Shurtleff SA, Downing JR, Behm FG, Harrison PL, Ribeiro RC, Pena O, Crist WM, Antillon FG (2000) Acute lymphoblastic leukemia in a developing country: preliminary results of a nonrandomized clinical trial in El Salvador. J Pediatr Hematol Oncol 22(6):495501

11. Magrath I, Shanta V, Advani S, Adde M, Arya LS, Banavali S, Bhargava M, Bhatia K, Gutiérrez M, Liewehr D, Pai S, Sagar TG, Venzon D (2005) Treatment of acute lymphoblastic leukaemia in countries with limited resources; lessons from use of a single protocol in India over a twenty year period. Eur $\mathrm{J}$ Cancer 41:1570-1583

12. Masera G, Baez F, Biondi A, Cavalli F, Conter V, Flores A, Fontana G, Fossati Bellani F, Lanfranco P, Malta A, Mendez G, Ocampo E, Pacheco C, Riva L, Sala A, Silva F, Sessa C, Tognoni G (1998) North-South twinning in paediatric haemato-oncology: the La Mascota programme, Nicaragua. Lancet 352:1293-1296

13. Temmim L, Baker H, Al-Jarallah MA, Manguno H, Madda JP, Sinowatz F (2004) Clinical characteristics and pathological classification of non-hodgkin's lymphoma in Kuwait. Results of a collaborative study with the International Lymphoma Study Group (ILSG). Leuk Lymphoma 45(9):1865-1871

14. Howard SC, Ribeiro RC, Pui CH (2005) Strategies to improve outcomes of children with cancer in low-income countries. Eur J Cancer 41(11):1584-1587

15. Eden $T$ (2002) Translation of cure for acute lymphoblastic leukaemia to all children. Br J Haematol 118:945-951

16. Greenberg PL, Gordeuk V, Issaragrisil S, Siritanaratkul N, Fucharoen S, Ribeiro PC (2001) Major hematologic diseases in the developing world - new aspects of diagnosis and management of thalassemia, malarial anemia, and acute leukemia. Hematology Am Soc Hematol Educ Program 479-498 